\title{
Appendix of Chinese names
}

\begin{tabular}{|c|c|}
\hline Ah Boon & 亚文 \\
\hline Ah Pek & 阿伯 \\
\hline Ang Ya Pek & 红爷伯 \\
\hline Anxi & 安溪 \\
\hline Anxi Chenghuangmiao & 安溪城隍庙 \\
\hline Anxi Chenghuangmiao fushu Yinfu Tan & 安溪城隍庙附属阴府坛 \\
\hline Anxi Dongyue Si Chenghuangmiao & 安溪东岳寺城隍庙 \\
\hline Ba Jiajiang & 八家将 \\
\hline Ba she chuan sai xiao diyu & 拔舌穿腮小地狱 \\
\hline $\mathrm{Ba} \mathrm{Ye}$ & 八爷 \\
\hline Ba Ye Gong & 八爷公 \\
\hline Bai Wuchang & 白无常 \\
\hline Bak Ya Pek & 北爷伯 \\
\hline Bao Bei Ya & 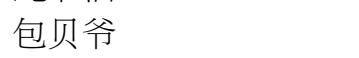 \\
\hline Bao feng bojue wu yi wushuang & 褒封伯爵五邑无双 \\
\hline Bao Gong & 包公 \\
\hline Beidi & 北帝 \\
\hline Beigang Chaotian Gong & 北港朝天宫 \\
\hline Biancheng Wang & 市城王 \\
\hline Cai Changyu & 蔡常毓 \\
\hline Chen & 陈 \\
\hline Chen Meiying & 陈美英 \\
\hline Chen Qixin & 陈其新 \\
\hline Chen Yiqun & 陈益群 \\
\hline Chenghuang & 城隍 \\
\hline Chengzong & 成宗 \\
\hline Choa Bao Bei Ya & 蔡包贝爷 \\
\hline Chong xi pao guan Bamin di yi & 宠锡袍冠八闽第一 \\
\hline Chujiang Wang & 楚江王 \\
\hline Chun & 春 \\
\hline Cixi & 慈禧 \\
\hline Da Bei Zhou & 大悲咒 \\
\hline
\end{tabular}


Da qige

Da Shi Ye

Dajia Zhenlan Gong

Daxie shen en xun Hei Bai Wuchang

Di Guan Dadi

Di Ya Pek

Difang Fu

Diji Gong

Diji Zhu

Ding shi li feng xiao diyu

Diyu Youji

Dizang pusa benyuan jing

Dizangwang (Bodhisattva)

Dong

Dongshiliao Dongan Gong

Dongyue Dadi

Dongyue $\mathrm{Si}$

Dongyue Si Chenghuangmiao

Doumu Gong

Doumu Niang-niang

Dushi Wang

Fan Di Ya

Fan Jiangjun

Fan Wujiu

Fazhu Gong

Fei dao huo shi diyu

Fengcheng

Fengdu

Fenglishan Tiangong Tan

Fo Shuo shiba ni li jing

Foshuo Dazang zhengjao xuepen jing

Fujian Province

Gan

Goh Yong Hau (Dato)

Guan Gong

Guan Sheng Dijun

Guangming Ribao

Guanyin

Gui Wang

Guo Dacheng

Hao Zhu Ya
大旗歌

大士爺

大甲镇澜宫

答谢神恩熏黑白无常

地官大帝

二爷伯

地方府

地基公

地基主

钉石立峰小地狱

地狱游记

地藏菩薩本願經

地藏王

冬

东势寮东安宫

东岳大帝

东岳寺

东岳寺城隍庙

斗母宫

斗母娘娘

都市王

范二爷

范将军

范无救

法主公

飞刀火石地狱

凤城

酆都

凤梨山天公坛

佛说十八泥犁经

佛说大藏正教血盆经

福建

干

吴学豪

关公

关圣帝君

光明日报

观音

鬼王

郭大诚

孝子爷 
232 APPENDIX OF CHINESE NAMES

Hei Bai Wuchang

Hei Ling Jiangjun

Hei Wuchang

Heishi Xiansheng

Hokkien

$\mathrm{Hu}$ Jingze

Huaguang

Huaguang Dadi

Huangquan

Jian shu diyu

Jianzi Gong

Jigong

Jin Shui Gang

Jiu Bao Bei Ya

Jiu Ye Gong

Jiucaiba Chenghuangmiao

Kang Di Ya

Kim Ya Pek

Kou yan xiao diyu

Kuomintang

Kuzhu qiao

Lai Dong Sen

Laisheng Gong

Lang Cia Ah Pek

Lang Pek

Lao You

Laojun

Laoquanan Zhangzhong Ban

Lee Huck Chye

Leigong

Li Shimin

Liangfu

Lim Bao Bei Ya

Lin

Lin Maosheng

Lin Yayuan

Lin Zhangshan

Lina

Lingbao

Lingguan Ma Yuanshuai

Linghai Dian
黑白无常

黑令将军

黑无常

黑石先生

闽南语

胡金泽

华光

华光大帝

黄泉

剑树地狱

目仔公

济公

汫水港

周包贝爷

九爷公

非菜芭城隍庙

江二爺

金爷伯

抠眼小地狱

国民党

苦竹桥

內东成

来圣宫

人车阿伯

人伯

老友

老君

老泉安掌中班

李学财

雷公

李世民

梁父

林包贝爷

林

林茂盛

林亚源

林长杉

丽娜

灵宝

灵官马元帅

灵海殿 
Liu

Liutian

Lo Qio Sian Pek

Longde Tang

Longhu Gong

Lu Xian Zan

Mamian

Mazu

Meng Po

Mile Pusa

Minnan

Minxiong Zhuang zongjiao tuanti taizhang

Muar Dasheng Gong Chenghuang Dian

Naihe (Bridge)

Nan Bei Dou Xingjun

Nankunshen

Nanlai Dian

Neicang Baibao jing

Nezha

Nie jingtai

Niutou

Nong xie diyu

Pai Gu Pek

Pangu Xianshi

Pangzu

Pingdeng Wang

Qi e'ah So

Qi Ye

Qian Bao Bei Ya

Qielan Pusa

Qingshui Zushi

Qingti

Qinguang Wang

Qingxi Chenghuang Xianyou Bozhu wenhua zhi ye

Qingyun Dian

Qio Pek

Qiu

Qiu Qiuxing

Sa Ya Pek

San Taizi
刘

六天

老笑仙伯

龙德堂

龙湖宫

炉香赞

马面

妈祖

孟婆

弥勒菩萨

闽南语

民雄庄宗教团体台帐

麻坡大圣宫城隍殿

奈何桥

南北斗星君

南鲲鰞

南莱殿

内藏百宝经

哪吒

藍镜台

牛头

脓血地狱

排骨伯

盘古仙师

旁足

平等王

青蚵仔嫂

七爷

千包贝爷

伽蓝菩萨

清水祖师

目連, 青提

秦廣王

清溪城隍显佑伯主文化之夜

清云殿

笑伯

秋

邱秋星

三爷伯

三太子 
Sanbao Gong

Sanguan

Sanqing Zushi

Santian

Sanzhong Gong

Shancai Tongzi

Shangdi

Shangqing

Shanlong Miao

She meiren

Sheliao Zinan Gong

Shengan Dian (Temple)

Shenglian Gong

Shenglin Gong

Shengxian Tang

Shensi bu

Shi

Shi Dian Da Er Ye Bo wenhua jiaoliu hui

Shi Dian Yenwang

Shiba nili jing

Shiba Wang Gong

Shiding Wu Lu Caishen Miao

Shiwang jing

Siming Panguan

Sin Hock Min (Foundation)

So

Songdi Wang

Songji Zhi Zha Dian

State of $\mathrm{Lu}$

Sun Wukong

Tai Shan

Tai shang ling bao ba du wang shan shishi keyi

Tai shang qing shen ke

Tai yi jiu ku miao jing

Taichung Guangtian Gong

Taipingjing

Taishan Fu Jun

Taishan Wang

Taisui

Taiyi Jiuku Tianzun
三宝宫

三官

三清祖师

三天

三中宫

善才童子

上帝

上清

善龙庙

蛇美人

社寮紫南宫

圣安殿

圣莲宫

圣林宫

圣贤堂

生死簿

施

十殿大二爷伯文化交流会

十殿閻王

十八泥犁經

十八王公

石碇五路财神庙

十王經

司命判官

申学明

苏

宋帝王

松记纸扎店

鲁

孙悟空

泰山

太上灵宝拔度望山施食科仪

太上請神科

太乙救苦妙经

台中广天宫

太平经

泰山府君

泰山王

太岁

太乙救苦天尊 
Tan

Tan Tua Lao

Tan Tua Ya

Tao Hongjing

Tiandeyuan Xunyin Fu

Tua Di Ya Pek

Tua Pek Kong Beo

Tua Pek Kong cian li to

Tua Pek Kung

Tua Ya Pek

Tudi Gong

Wang Chengfa

Wang Wenjiu

Wang Yijie

Wangsi Cheng

Wangye

Wanshan Ye

Weiling Gong

Wu Dan Zhou

Wu Fang Gui Di

Wu Jing

Wu She Chenghuang Gong

Wu Yiming

Wuguan Wang

Wulu Caishen

Xia

Xiao Fo

Xiao Tianping

$\mathrm{Xie}$

Xie Bian

Xie Jiangjun

Xie Liwen

Xin Jing

Xinjiawang Chenghuangmiao

Xiude Foshi Tuan

Xiwangmu

Xu Xiaoshan

Xuanhuang Dian

Xuantian Shangdi

Xunyin Miao

Yang
陈

陳大炮

陈 大爷

陶弘景

天德园巡阴府

大二爷伯

大伯公庙

大伯公千字图

大伯公

大爷伯

土地公

王琛发

王文九

王益杰

枉死城

王爷

万善爷

威灵公

五丹咒

五方鬼帝

五经

五舍城隍公

吴易铭

五官王

五路财神

夏

笑佛

萧天平

谢

谢必安

谢将军

谢礼文

心经

麻坡新加望城隍庙

修德佛事团

西王母

许晓山

玄皇殿

玄天上帝

巡阴庙

楊 
Yang Zanru

Yangzhou Jing Shui

Yanluo Wang

Yaozhan xiao diyu

Yilan Jinxing Gong

Yinfu Tan

Yinyuan jing

Yong Mun Tong (Dato)

You guo diyu

Yu Feng Nan Fu Xuanshan Miao

Yuanshi tianzun jidu xiehu zhengjing

Yulanpen (Sutra)

Yuli chao chuan

Yunshan Dian

Yushan Temple

Zai Ya Pek

Zeng

Zeng Zhongying

Zhang Dan

Zhang Daoling

Zhang Songba

Zhang Wuwen

Zhao Gongming

Zheng $\mathrm{He}$

Zhengao

Zhenling weiye tu

Zhenlinshan Jieyuan Tang Chenghuangmiao

Zhenren Gong

Zhong Kui

Zhongliao Anxi Chenghuangmiao

Zhongyuan

Zhongyuan Dadi

Zhu Sheng Niang-niang

Zhuangzi

Zhuanlun Wang
杨赞儒

杨枝净水赞

阎罗王

腰斩小地狱

宜兰进兴宫

阴府坛

因缘经

楊瞞冬

油锅地狱

玉封南府玄善庙

元始天尊济度血湖真经

孟兰盆

玉历钞传

云善殿

玉善殿

财爷伯

曾

曾仲影

张旦

张道陵

张松峇

张乌文

赵公明

郑和

真誥

真灵位业图

振林山结缘堂城隍庙

真人宫

钟馗

中寮安溪城隍庙

中元

中元大帝

註生娘娘

庄子

转轮王 